\title{
Cognitive behavioural therapy was effective for adolescent depression after controlling for adverse predictors of outcome
}

\author{
Brent DA, Kolko DJ, Birmaher B, et al. Predictors of treatment efficacy in a clinical trial of three psychosocial treatments for \\ adolescent depression.J Am Acad Child Adolesc Psychiatry 1998 Sep;37:906-14.
}

\section{Question}

In adolescents with depression, what are the predictors of treatment outcome and of differential treatment response when evaluating the effectiveness of 3 psychosocial therapies?

\section{Design}

Randomised controlled trial with follow up to the end of treatment (range 12-16 weeks).

\section{Setting}

Western Psychiatric Institute and Clinic, Pittsburgh, Pennsylvania, USA.

\section{Patients}

107 adolescent outpatients 13-18 years of age with a DSM-III-R diagnosis of major depression and a Beck Depression Inventory (BDI) score $\geqslant 13$. Exclusion criteria were psychotic, bipolar, or obsessive-compulsive disorder; eating disorder; substance abuse; or ongoing physical or sexual abuse.

\section{Intervention}

Patients were allocated to 1 of 3 manual based, brief (12-16 sessions) psychosocial treatments: cognitive behavioural therapy (CBT), systemic behavioural family therapy (SBFT), or nondirective supportive therapy (NST).

\section{Main outcome measures}

Presence of DSM-III-R major depressive disorder at the end of treatment, remission (defined as absence of major depressive disorder at the end of treatment and a BDI score $<9$ for $\geqslant 3$ consecutive sessions sustained until the end of treatment), and presence of functional impairment ( $<60$ on the Children's Global Assessment Scale at the end of treatment).

\section{Main results}

Analysis was by intention to treat. Continued depression at the end of treatment was predicted by clinical referral (versus by advertisement), comorbid anxiety disorder at intake, and higher levels of cognitive distortion and hopelessness at intake. Patients with comorbid anxiety responded better to CBT than to SBFT and NST. 3 factors predicted failure to achieve clinical remission: a high level of initial self reported depression, a higher level of cognitive distortion, and higher hopelessness scores. Taking these factors into consideration, patients in the NST group were 3.1 times (95\% CI 1.1 to 4.7 ) more likely to have no recovery at the end of treatment than those who received CBT. The efficacy of CBT deteriorated in the presence of high maternal depressive symptoms. Poor functional status at the end of treatment was predicted by high interview rated depression level at intake. Taking this factor into consideration did not lead to statistically significant differences between treatments. CBT was still superior to NST and SBFT when controlling for multiple adverse predictors and the effectiveness of CBT did not deteriorate with increasing numbers of adverse predictors.

\section{Conclusions}

In adolescents with major depression, poor treatment response was predicted by comorbid anxiety disorder, clinical referral, and greater severity of depression. Patients with comorbid anxiety responded better to CBT than to the other treatments. The efficacy of this type of intervention decreased in the face of maternal depressive symptoms but not in the presence of multiple adverse predictors.

Source of funding: National Institute of Mental Health.

For correspondence: Dr D A Brent, Western Psychiatric Institute and Clinic, 3811 O'Hara Street, Pittsburgh, PA 15213, USA. Fax +1 4126247997.

\section{Commentary}

Depression in young people is a problem with such pervasive features that one can find abnormalities in almost any domain (eg, cognitive, family) to justify any treatment. ${ }^{1}$ A great variety of psychosocial interventions have therefore been used with depressed children, including CBT, psychotherapy, and family therapy. Until recently, however, little was known about the relative efficacy of these treatments.

This is the second paper from Brent $e t$ al's well planned trial comparing CBT, family therapy, and non-directive supportive therapy in adolescents with major depression. The first paper reported that CBT was superior to the other interventions. ${ }^{2}$ In this second paper, the issue of who responds best to treatment is addressed. The issue is important because although everyone agrees that depression among the young is a highly heterogeneous problem, we have few clues about who responds best to treatment.

The results show that a poor outcome was predicted by various factors, particularly the source of recruitment to the study - that is, patients referred through the clinic had a worse outcome than patients recruited through advertisements. Other predictors included hopelessness and a higher level of cognitive distortion at the start of treatment. CBT seemed to be less effective when the mother was depressed but otherwise its efficacy was not diminished by the presence of multiple adverse predictors of outcome.

What, then, are the clinical implications of these findings? The first is that in future it cannot be assumed that results of trials with non-clinical samples will apply to patients referred to psychiatric clinics. Secondly, CBT is not differentially more effective in patients with high levels of cognitive distortion. The beneficial effects of CBT may not therefore be mediated by changes in negative cognitions. Thirdly, however, CBT continues to work better than alternative treatments in patients with adverse prognostic factors.

Richard C Harrington, MD Royal Manchester Children's Hospital Manchester, UK

1 Harrington RC, Whittaker J, Shoebridge P. Psychological treatment of depression in children and adolescents: a review of treatment research. Br J Psychiatry 1998;173:291-8

2 Brent D, Holder D, Kolko D, et al. A clinical psychotherapy trial for adolescent depression comparing cognitive, family, and supportive treatments. Arch Gen Psychiatry 1997;54:87785. 